\title{
Endoscopic ultrasound-guided choledochojejunost- omy with a lumen-apposing metal stent: a shortcut for biliary drainage
}

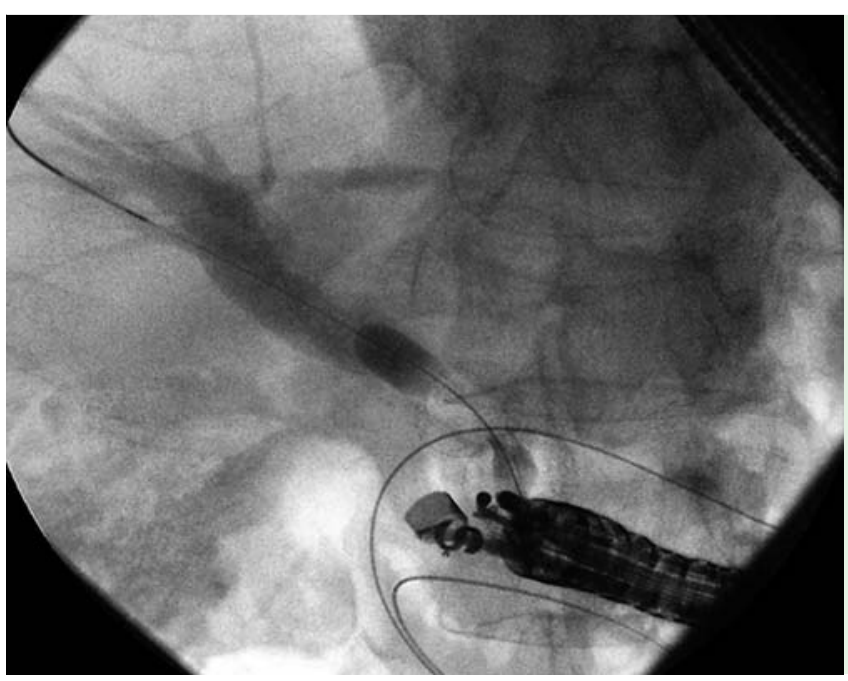

Fig. 1 Fluoroscopic image of a lumenapposing metal stent with the proximal end in the common bile duct and the distal end in the jejunum, placed for biliary drainage in a 64-year-old man who had gastric cancer and a Billroth II anastomosis. The patient presented with cholangitis and biliary obstruction due to tumor recurrence.

Endoscopic-ultrasound (EUS)-guided biliary drainage is a therapeutic option for patients with biliary pathology and altered gastrointestinal anatomy in whom conventional endoscopic retrograde cholangiopancreatography (ERCP) has failed [1 3]. We present the case of a patient undergoing EUS-guided choledochojejunostomy with a fully covered, lumen-apposing metal stent $(\bullet$ Video 1 ).

A 64-year-old man who had gastric cancer and a Billroth II anastomosis presented with cholangitis and biliary obstruction due to tumor recurrence. Conventional ERCP and single-balloon ERCP were unsuccessful. EUS-guided biliary drainage was performed. The echoendoscope (GF-

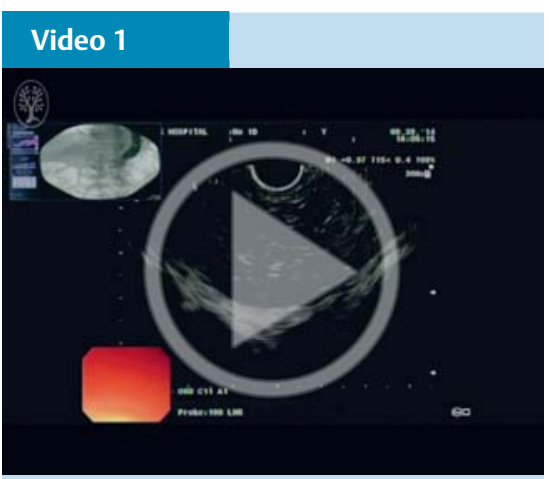

Creation of an endoscopic ultrasound-guided choledochojejunostomy with a lumen-apposing metal stent as a shortcut for biliary drainage.
UCT180; Olympus, Central Valley, Pennsylvania, USA) identified the left intrahepatic duct. A 19-gauge needle (ECHO 19 EchoTip; Cook Medical, Winston-Salem, North Carolina, USA) was used to access the duct. Cholangiography revealed a distal biliary stricture with upstream dilatation but nondilated intrahepatic ducts. Contrast extended to the ampulla but did not pass into the small bowel.

Attempts to pass a guidewire (Hydra Jagwire; Boston Scientific, Natick, Massachusetts, USA) into the duct were unsuccessful because of insufficiently dilated left intrahepatic duct radicals. The echoendoscope was advanced to the jejunum adjacent to the opacified extrahepatic bile duct. The duct was accessed with a 19-gauge needle, a wire was advanced, and a dilating balloon (Hurricane RX Biliary Balloon Dilation Catheter; Boston Scientific) was used to create a fistulous tract. A 10-mm lumen-apposing metal stent (Axios; Xlumena, Mountain View, California, USA) was deployed with the proximal end in the extrahepatic bile duct and the distal end in the jejunum ( $\bullet$ Fig. 1 ). A balloon dilator (CRE; Boston Scientific) was used to dilate the stent. Two doublepigtail plastic stents (Advanix; Boston Scientific) were deployed to separate the lumen-apposing metal stent from the opposite wall of the duct ( $\bullet$ Fig. 2 ).

The patient's bilirubin level decreased, and he was discharged from the hospital.

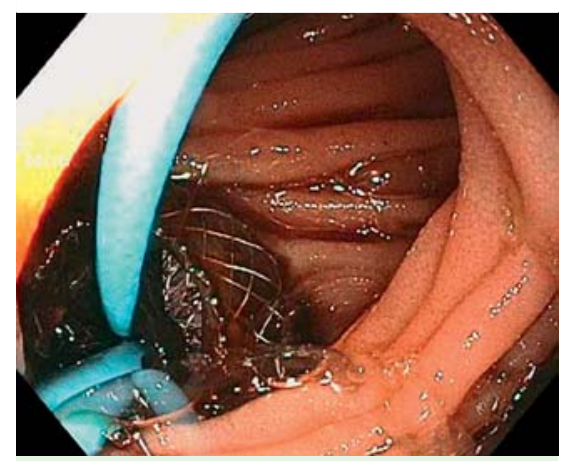

Fig. 2 Endoscopic view of the lumen-apposing metal stent with the proximal end in the common bile duct and the distal end in the jejunum. A double-pigtail plastic stent is used to anchor the position.

At 3- and 6-month follow-up, his serum liver chemistries remained normal, and imaging confirmed a stable stent position with resolution of the ductal dilatation. The creation of an EUS-guided choledochojejunostomy with a lumen-apposing metal stent for biliary decompression offers a minimally invasive option for patients with altered anatomy who require ERCP.

\section{Endoscopy_UCTN_Code_TTT_1AS_2AD}

Competing interests: Michel Kahaleh, MD, has received grant support from Boston Scientific, Fujinon, EMcison, Xlumena Inc., W.L. Gore, MaunaKea, Apollo Endosurgery, Cook Endoscopy, ASPIRE Bariatrics, GI Dynamics, NinePoint Medical, Merit Medical and MI Tech. He is a consultant for Boston Scientific, Xlumena Inc., Concordia Laboratories Inc. and MaunaKea Tech.

\section{Amy Tyberg, Kunal Karia, Steven Zerbo, Reem Z. Sharaiha, Michel Kahaleh}

Department of Gastroenterology and Hepatology, Weill Cornell Medical College, New York, New York, USA 


\section{References}

1 Perez-Miranda M, De la Serna Higuera C, GilSimon $P$ et al. EUS-guided choledochoduodenostomy with lumen-apposing metal stent after failed rendezvous in synchronous malignant biliary and gastric outlet obstruction (with video). Gastrointest Endosc 2014; 80: 342; discussion 343-344

2 Itoi T, Binmoeller KF. EUS-guided choledochoduodenostomy by using a biflanged lumen-apposing metal stent. Gastrointest Endosc 2014; 79: 715

3 Nicholson JA, Johnstone M, Raraty MGT et al. Endoscopic ultrasound-guided choledochoduodenostomy as an alternative to percutaneous trans-hepatic cholangiography. HPB (Oxford) 2012; 17: 483-486
Bibliography

DOI http://dx.doi.org/

10.1055/s-0034-1392318

Endoscopy 2015; 47: E342-E343

(c) Georg Thieme Verlag KG

Stuttgart · New York

ISSN 0013-726X
Corresponding author

Michel Kahaleh, MD

Weill Cornell Medical College

Department of Gastroenterology and Hepatology 1305 York Avenue

4th Floor

New York, NY 10021

USA

Fax: +1-646-962-0110

mkahaleh@gmail.com 